JOURNAL OF

SYMPLECTIC GEOMETRY

Volume 6, Number 2, 127-138, 2008

\title{
HOLOMORPHIC VECTOR FIELDS AND PERTURBED EXTREMAL KÄHLER METRICS
}

\author{
Akito Futaki
}

\begin{abstract}
We prove a theorem which asserts that the Lie algebra of all holomorphic vector fields on a compact Kähler manifold with a perturbed extremal metric has the structure similar to the case of an unperturbed extremal Kähler metric proved by Calabi.
\end{abstract}

\section{Introduction}

Let $M$ be a compact symplectic manifold with symplectic form $\omega$. On the space $\mathcal{J}$ of all $\omega$-compatible complex structures $J$, there is a natural symplectic form with respect to which the scalar curvature $S(J)$ of the Kähler manifold $(M, \omega, J)$ becomes a moment map for the action of the group of all Hamiltonian diffeomorphisms of $(M, \omega)$ acting on $\mathcal{J}$ (c.f. $[\mathbf{3}, \mathbf{4}]$ ). This means that the problem of finding extremal Kähler metrics can be set in the framework of stability in the sense of geometric invariant theory. It was shown in [7] that, perturbing the symplectic form on $\mathcal{J}$ and the scalar curvature incorporating with the higher Chern classes and with a small real parameter $t$, the perturbed scalar curvature $S(J, t)$ becomes a moment map with respect to the perturbed symplectic form on $\mathcal{J}$. Note that the unperturbed scalar curvature is the trace of the first Chern class, see Section 2 for the precise definitions.

Recall that a Kähler metric $g$ is called an extremal Kähler metric if the $(1,0)$-part of the gradient vector field of the scalar curvature $S$

$$
\operatorname{grad}^{\prime} S=g^{i \bar{j}} \frac{\partial S}{\partial \bar{z}^{j}} \frac{\partial}{\partial z^{i}}
$$

is a holomorphic vector field. Extremal Kähler metrics are critical points of two functionals. One is the so-called Calabi functional. This is a functional $\Psi$ on the space $\mathcal{K}_{\omega_{0}}$ of all Kähler forms in a fixed de Rham class $\omega_{0}$ with 
fixed complex structure $J$. If $\omega \in K_{\omega_{0}}$ and $S(\omega)$ denotes the scalar curvature of $\omega$, then

$$
\Psi(\omega)=\int_{M} S(\omega)^{2} \omega^{m}
$$

where $m=\operatorname{dim}_{\mathbb{C}} M$. Calabi originally defined extremal Kähler metrics to be the critical points of $\Psi$. The other functional $\Phi$ is defined on $\mathcal{J}$. If $S(J)$ denotes the scalar curvature of the Kähler manifold $(M, \omega, J)$ for $J \in \mathcal{J}$, then

$$
\Phi(J)=\int_{M} S(J)^{2} \omega^{m}
$$

It is easy to see that the extremal Kähler metrics are exactly the critical points of $\Phi$ from the fact that the scalar curvature is the moment map on $\mathcal{J}$ for the action of Hamiltonian diffeomorphisms as mentioned above.

Inspired by a work of Bando [1] the author defined in [7] perturbed extremal Kähler metrics as follows: the Kähler metric $g$ for $(M, \omega, J)$ is called a perturbed extremal Kähler metric if the (1,0)-part of the gradient vector field

$$
\operatorname{grad}^{\prime} S(J, t)=g^{i \bar{j}} \frac{\partial S(J, t)}{\partial \bar{z}^{j}} \frac{\partial}{\partial z^{i}}
$$

is a holomorphic vector field. From the fact that $S(J, t)$ becomes a moment map on $\mathcal{J}$ with respect to the perturbed symplectic structure, one can see that the critical points of the functional

$$
\Phi(J)=\int_{M} S(J, t)^{2} \omega^{m}
$$

are $J$ 's for which the Kähler metric of $(M, \omega, J)$ is a perturbed extremal Kähler metric. A computation in Remark 3.3 in [7] shows that it is not clear if perturbed extremal Kähler metrics are the critical points of $\Psi(\omega)=$ $\int_{M} S(\omega, t)^{2} \omega^{m}$.

In $[\mathbf{1 0}]$, X. Wang explains how one gets the decomposition theorem of Calabi [2] for the structure of the Lie algebra of all holomorphic vector fields on compact Kähler manifolds with extremal Kähler metrics in the finite dimensional setting of the framework of the moment maps, see also [6]. On the other hand, L. Wang [9] explains how one gets the Hessian formulae for the Calabi functional and the functional $\Phi$ in the finite dimensional setting of the framework of moment maps. Recall that the Hessian formula for the Calabi functional plays the key role for the proof of Calabi's decomposition theorem of the Lie algebra of all holomorphic vector fields on compact Kähler manifolds with extremal Kähler metrics. Because of the above mentioned difference between the perturbed case and the unperturbed case, one can not expect that the same proof as the unperturbed case by Calabi can be applied to the perturbed case. The purpose of this paper is to see L. Wang's finite dimensional arguments provide us a rigorous proof 
of Calabi's decomposition theorem for compact Kähler manifolds with perturbed extremal Kähler metrics. Thus we obtain a similar statement of the decomposition theorem:

Theorem 1.1. Let $M$ be a compact Kähler manifold with a perturbed extremal Kähler metric. Let $\mathfrak{h}(M)$ be the Lie algebra of all holomorphic vector fields and $\mathfrak{k}$ be the real Lie algebra of all Killing vector fields of $M$. Then

(a) $\mathfrak{h}_{0}(M):=\mathfrak{k} \otimes \mathbb{C}$ is the maximal reductive subalgebra of $\mathfrak{h}(M)$.

(b) The (1,0)-part of the gradient vector field

$$
\operatorname{grad}^{\prime} S(J, t)=g^{i \bar{j}} \frac{\partial S(J, t)}{\partial \bar{z}^{j}} \frac{\partial}{\partial z^{i}}
$$

of $S(J, t)$ belongs to the center of $\mathfrak{h}_{0}(M)$.

(c) $\mathfrak{h}(M)$ has the structure of semi-direct decomposition

$$
\mathfrak{h}(M)=\mathfrak{h}_{0}(M)+\sum_{\lambda \neq 0} \mathfrak{h}_{\lambda}(M),
$$

where $\mathfrak{h}_{\lambda}(M)$ is the $\lambda$-eigenspace of the adjoint action of $\operatorname{grad}^{\prime} S(J, t)$.

The proof of this theorem is given by following the arguments of L. Wang almost word for word.

One may try to prove the existence of perturbed extremal Kähler metrics by extending known results for the unperturbed extremal Kähler metrics. As for the vector bundle case, Leung [8] has proved the existence of a kind of perturbed Hermitian-Einstein equation derived from an idea of the moment map which is related to Gieseker stability.

Throughout this paper, Hermitian inner products are anti-linear in the first component and linear in the second component.

\section{Perturbed extremal Kähler metric}

Let $M$ be a compact symplectic manifold of dimension $2 m$ with symplectic form $\omega, \mathcal{J}$ the space of all $\omega$-compatible complex structures on $M$. Then for each $J \in \mathcal{J},(M, J, \omega)$ becomes a Kähler manifold. For a pair $(J, t), t$ being a small real number, we define a smooth function $S(J, t)$ on $M$ by

$$
S(J, t) \omega^{m}=c_{1}(J) \wedge \omega^{m-1}+t c_{2}(J) \wedge \omega^{m-2}+\cdots+t^{m-1} c_{m}(J),
$$

where $c_{i}(J)$ is the $i$-th Chern form defined by

$$
\operatorname{det}\left(I+\frac{i}{2 \pi} t \Theta\right)=1+t c_{1}(J)+\cdots+t^{m} c_{m}(J),
$$

$\Theta$ being the curvature form of the Kähler manifold $(M, J, \omega)$. Note that we use $S(J, t)$ in place of $S(J, T) / 2 m \pi$ in [7] to avoid the clumsy constant $1 / 2 m \pi$. 
Definition 2.1. The Kähler metric $g$ of the Kähler manifold $(M, J, \omega)$ is called a $t$-perturbed extremal Kähler metric or simply perturbed extremal metric if

$$
\operatorname{grad}^{\prime} S(J, t)=\sum_{i, j=1}^{m} g^{i \bar{j}} \frac{\partial S(J, t)}{\partial \bar{z}^{j}} \frac{\partial}{\partial z^{i}}
$$

is a holomorphic vector field.

The following was proved in [7, Proposition 3.2].

Proposition 2.2. The critical points of the functional $\Phi$ on $\mathcal{J}$ defined by

$$
\Phi(J)=\int_{M} S(J, t)^{2} \omega^{m}
$$

are the perturbed extremal Kähler metrics.

The proof of this proposition essentially follows from the fact that the perturbed scalar curvature $S(J, t)$ gives the moment map for the action of the group of Hamiltonian diffeomorphisms with respect to a perturbed symplectic structure on $\mathcal{J}$. This perturbed symplectic structure is described as follows. The tangent space of $\mathcal{J}$ at $J$ is identified with a subspace of $C^{\infty}\left(\operatorname{Sym}\left(\otimes^{2} T^{\prime \prime *} M\right)\right)$ of all smooth sections of $\operatorname{Sym}\left(\otimes^{2} T^{\prime \prime *} M\right)$. For a small real number $t$, we define an Hermitian structure on $C^{\infty}\left(\operatorname{Sym}\left(\otimes^{2} T^{\prime \prime *} M\right)\right)$ by

$$
\begin{aligned}
(\nu, \mu)_{t}= & \int_{M} m c_{m}\left(\bar{\nu}_{j k} \mu^{i} \bar{\ell} \frac{\sqrt{-1}}{2 \pi} d z^{k} \wedge d \overline{z^{\ell}}, \omega \otimes I\right. \\
& \left.+\frac{\sqrt{-1}}{2 \pi} t \Theta, \ldots, \omega \otimes I+\frac{\sqrt{-1}}{2 \pi} t \Theta\right)
\end{aligned}
$$

for $\mu$ and $\nu$ in the tangent space $T_{J} \mathcal{J}$, where $c_{m}$ is the polarization of the determinant viewed as a $G L(m, \mathbb{C})$-invariant polynomial, i.e., $c_{m}\left(A_{1}, \ldots\right.$, $\left.A_{m}\right)$ is the coefficient of $m ! t_{1} \cdots t_{m}$ in $\operatorname{det}\left(t_{1} A_{1}+\cdots+t_{m} A_{m}\right)$, where $I$ denotes the identity matrix and $\Theta=\bar{\partial}\left(g^{-1} \partial g\right)$ is the curvature form of the Levi-Civita connection, and where $u_{j k} \mu_{l}^{i}$ should be understood as the endomorphism of $T_{J}^{\prime} M$ which sends $\partial / \partial z^{j}$ to $u_{j k} \mu_{\bar{l}}^{i} \partial / \partial z^{i}$. When $t=0,(2.5)$ gives the usual $L^{2}$-inner product. The perturbed symplectic form $\Omega_{J, t}$ at $J \in \mathcal{J}$ is then given by

$$
\begin{aligned}
\Omega_{J, t}(\nu, \mu)= & \Re(\nu, \sqrt{-1} \mu)_{t} \\
= & \Re \int_{M} m c_{m}\left(\bar{\nu}_{j k} \sqrt{-1} \mu \frac{\sqrt{-1}}{2 \pi} d z^{k} \wedge d \overline{z^{\ell}}, \omega \otimes I\right. \\
& \left.+\frac{\sqrt{-1}}{2 \pi} t \Theta, \ldots, \omega \otimes I+\frac{\sqrt{-1}}{2 \pi} t \Theta\right)
\end{aligned}
$$

where $\Re$ means the real part. In [7] we proved the following: 
Theorem 2.3. [7] If $\delta J=\mu$, then

$$
\delta \int_{M} u S(J, t) \omega^{m}=\Omega_{J, t}\left(2 \sqrt{-1} \nabla^{\prime \prime} \nabla^{\prime \prime} u, \mu\right) .
$$

Namely, the perturbed scalar curvature $S(J, t)$ gives a moment map with respect to the perturbed symplectic form $\Omega_{J, t}$ for the action of the group of Hamiltonian diffeomorphisms on $\mathcal{J}$.

Now we can prove Proposition 2.2. From (2.7) we have

$$
\begin{aligned}
\delta \int_{M} S(J, t)^{2} \omega^{m} & =2 \int_{M} S(J, t) \delta S(J, t) \omega^{m} \\
& =2 \Omega_{J, t}\left(2 \sqrt{-1} \nabla^{\prime \prime} \nabla^{\prime \prime} S(J, t), \mu\right) .
\end{aligned}
$$

This shows that $J$ is a critical point if and only if

$$
\nabla^{\prime \prime} \operatorname{grad}^{\prime} S(J, t)=0,
$$

i.e., the Kähler metric of $(M, \omega, J)$ is a perturbed extremal Kähler metric.

Let $\mathfrak{g}$ be the complexification of the Lie algebra of the group of Hamiltonian diffeomorphisms. Then $\mathfrak{g}$ is simply the set of all complex valued smooth functions $u$ with the normalization

$$
\int_{M} u \omega^{m}=0
$$

with the Lie algebra structure given by the Poisson bracket. The infinitesimal action of $u$ on $\mathcal{J}$ is given by $2 i \nabla^{\prime \prime} \nabla^{\prime \prime} u$, see Lemma 10 in [3] or Lemma 2.3 in [7]. Define $L: C^{\infty}(M) \otimes \mathbb{C}(\cong \mathfrak{g}) \rightarrow C^{\infty}(M) \otimes \mathbb{C}$ by

$$
\begin{aligned}
(v, L u)_{L^{2}}= & \left(\nabla^{\prime \prime} \nabla^{\prime \prime} v, \nabla^{\prime \prime} \nabla^{\prime \prime} u\right)_{t} \\
= & \int_{M} m c_{m}\left(\bar{v}_{j k} u_{\bar{\ell}}^{i} \frac{\sqrt{-1}}{2 \pi} d z^{k} \wedge d \overline{z^{\ell}}, \omega \otimes I\right. \\
& \left.+\frac{\sqrt{-1}}{2 \pi} t \Theta, \ldots, \omega \otimes I+\frac{\sqrt{-1}}{2 \pi} t \Theta\right) .
\end{aligned}
$$

More explicitly $L$ is expressed as

$$
\begin{aligned}
L u= & m c_{m}\left(u_{\bar{\ell} j k}^{i} \frac{\sqrt{-1}}{2 \pi} d z^{k} \wedge d \overline{z^{\ell}}, \omega \otimes I\right. \\
& \left.+\frac{\sqrt{-1}}{2 \pi} t \Theta, \cdots, \omega \otimes I+\frac{\sqrt{-1}}{2 \pi} t \Theta\right) / \omega^{m} .
\end{aligned}
$$


We define $\bar{L}: C^{\infty}(M) \otimes \mathbb{C} \rightarrow C^{\infty}(M) \otimes \mathbb{C}$ by $\bar{L} u:=\bar{L} \bar{u}$. Then $\bar{L}$ satisfies

$$
\begin{aligned}
(v, \bar{L} u)_{L^{2}}= & \left(\nabla^{\prime \prime} \nabla^{\prime \prime} \bar{u}, \nabla^{\prime \prime} \nabla^{\prime \prime} \bar{v}\right)_{t} \\
= & \int_{M} m c_{m}\left(u_{j k} \bar{v}^{i} \frac{\sqrt{-1}}{2 \pi} d z^{k} \wedge d \overline{z^{\ell}}, \omega \otimes I\right. \\
& \left.+\frac{\sqrt{-1}}{2 \pi} t \Theta, \ldots, \omega \otimes I+\frac{\sqrt{-1}}{2 \pi} t \Theta\right) .
\end{aligned}
$$

and

$$
\begin{aligned}
\bar{L} u= & m c_{m}\left(u_{j k}{ }^{i} \bar{\ell} \frac{\sqrt{-1}}{2 \pi} d z^{k} \wedge d \overline{z^{\ell}}, \omega \otimes I\right. \\
& \left.+\frac{\sqrt{-1}}{2 \pi} t \Theta, \cdots, \omega \otimes I+\frac{\sqrt{-1}}{2 \pi} t \Theta\right) / \omega^{m} .
\end{aligned}
$$

Lemma 2.4. If $v$ is a real smooth function and $\delta J=\nabla^{\prime \prime} \nabla^{\prime \prime} v$, then

$$
\delta S(J, t)=L v+\bar{L} v .
$$

Proof. Let $u$ be also a real smooth function. Then by (2.7)

$$
\begin{aligned}
\int_{M} u \delta S(J, t) \omega^{m} & =\Re\left(2 \sqrt{-1} \nabla^{\prime \prime} \nabla^{\prime \prime} u, \sqrt{-1} \mu\right)_{t} \\
& =\left(\nabla^{\prime \prime} \nabla^{\prime \prime} u, \nabla^{\prime \prime} \nabla^{\prime \prime} v\right)_{t}+\left(\nabla^{\prime \prime} \nabla^{\prime \prime} v, \nabla^{\prime \prime} \nabla^{\prime \prime} u\right)_{t} \\
& =(u, L v)_{L^{2}}+(u, \bar{L} v)_{L^{2}} .
\end{aligned}
$$

Lemma 2.5. Let $u$ and $v$ be real smooth functions and put $\mathcal{X}_{u}=2 \sqrt{-1} \nabla^{\prime \prime} \nabla^{\prime \prime} u$ and $\mathcal{X}_{v}=2 \sqrt{-1} \nabla^{\prime \prime} \nabla^{\prime \prime} v$. Then we have

$$
\Omega_{J, t}\left(\mathcal{X}_{u}, \mathcal{X}_{v}\right)=(\{u, v\}, S(J, t))_{L^{2}} .
$$

Proof. Consider $\mathcal{X}_{u}$ and $\mathcal{X}_{v}$ as the infinitesimal action of real Hamiltonian functions $u$ and $v$ on $\mathcal{J}$. Since $S(J, t)$ gives an equivariant moment map

$$
\int_{M} u S(\sigma J, t) \omega^{m}=\int_{M}\left(\sigma^{-1 *} u\right) S(J, t) \omega^{m}
$$

for a Hamiltonian diffeomorphism $\sigma$. If $\sigma$ is generated by the Hamiltonian vector field of a Hamiltonian function $v$, then (2.7) and (2.12) show

$$
\Omega_{J, t}\left(2 \sqrt{-1} \nabla^{\prime \prime} \nabla^{\prime \prime} u, 2 \sqrt{-1} \nabla^{\prime \prime} \nabla^{\prime \prime} v\right)=-\int_{M} S(J, t)\{v, u\} \omega^{m} .
$$

Lemma 2.6. For any smooth complex valued function $u$, we have

$$
(\bar{L}-L) u=-\frac{1}{2}\left(S(J, t)^{\alpha} u_{\alpha}-u^{\alpha} S(J, t)_{\alpha}\right),
$$

where $z^{\alpha}$ 's are local holomorphic coordinates and $u^{\alpha}=g^{\alpha \bar{\beta}} \partial u / \partial z^{\bar{\beta}}$, thus the right hand side being equal to $i\{u, S(J, t)\} / 2$. 
Proof. It is sufficient to prove when $u$ is a real valued function. Let $v$ be also a real valued smooth function. From (2.10) and (2.11), we have

$$
\begin{aligned}
(v, \bar{L} u-L u)_{L^{2}} & =\left(\nabla^{\prime \prime} \nabla^{\prime \prime} u, \nabla^{\prime \prime} \nabla^{\prime \prime} v\right)_{t}-\left(\nabla^{\prime \prime} \nabla^{\prime \prime} v, \nabla^{\prime \prime} \nabla^{\prime \prime} u\right)_{t} \\
& =\overline{\left(\nabla^{\prime \prime} \nabla^{\prime \prime} v, \nabla^{\prime \prime} \nabla^{\prime \prime} u\right)_{t}}-\left(\nabla^{\prime \prime} \nabla^{\prime \prime} v, \nabla^{\prime \prime} \nabla^{\prime \prime} u\right)_{t} .
\end{aligned}
$$

It follows from this that

$$
\begin{aligned}
2 \Re\left(\nabla^{\prime \prime} \nabla^{\prime \prime} v, i \nabla^{\prime \prime} \nabla^{\prime \prime} u\right)_{t} & =i\left(\nabla^{\prime \prime} \nabla^{\prime \prime} v, \nabla^{\prime \prime} \nabla^{\prime \prime} u\right)_{t}+\overline{i\left(\nabla^{\prime \prime} \nabla^{\prime \prime} v, \nabla^{\prime \prime} \nabla^{\prime \prime} u\right)_{t}} \\
& =-i(v,(\bar{L}-L) u)_{L^{2}} .
\end{aligned}
$$

Let $X_{u}$ denote the Hamiltonian vector field of $u$ : $i\left(X_{u}\right) \omega=d u$. Then $X_{u}=J$ $\operatorname{grad} u$ and $\{u, S\}=X_{u} S$. It then follows that

$$
\begin{aligned}
(v,(\bar{L}-L) u)_{L^{2}} & =2 i \Re\left(\nabla^{\prime \prime} \nabla^{\prime \prime} v, i \nabla^{\prime \prime} \nabla^{\prime \prime} u\right)_{t} \\
& =\frac{i}{2} \Re\left(\mathcal{X}_{v}, i \mathcal{X}_{u}\right)=\frac{i}{2} \Omega_{J, t}\left(\mathcal{X}_{v}, \mathcal{X}_{u}\right) \\
& =-\frac{i}{2}(\{u, v\}, S(J, t))_{L^{2}}=\frac{i}{2}(v,\{u, S(J, t)\})_{L^{2}} \\
& =\frac{i}{2}\left(v, X_{u} S(J, t)\right)_{L^{2}}=\frac{i}{2} \omega\left(v, g\left(X_{u}, J \operatorname{grad} S(J, t)\right)\right)_{L^{2}} \\
& =\frac{i}{2}(v, d u(J \operatorname{grad} S(J, t)))_{L^{2}} \\
& =-\frac{1}{2}\left(v, S(J, t)^{\alpha} u_{\alpha}-u^{\alpha} S(J, t)_{\alpha}\right)_{L^{2}} .
\end{aligned}
$$

Lemma 2.7. Let $u$ be a real smooth function and suppose $\delta J=\nabla^{\prime \prime} \nabla^{\prime \prime} u$. Then

$$
\delta \int_{M} S(J, t)^{2} \omega^{m}=4(u, L S(J, t))_{L^{2}}=4(u, \bar{L} S(J, t))_{L^{2}} .
$$

Proof. By (2.8)

$$
\begin{aligned}
\delta \int_{M} S(J, t)^{2} \omega^{m} & =2 \Omega_{J, t}\left(2 i \nabla^{\prime \prime} \nabla^{\prime \prime} S(J, t), \nabla^{\prime \prime} \nabla^{\prime \prime} u\right) \\
& =4 \Re\left(\nabla^{\prime \prime} \nabla^{\prime \prime} S(J, t), \nabla^{\prime \prime} \nabla^{\prime \prime} u\right)_{t} \\
& =2\left(\nabla^{\prime \prime} \nabla^{\prime \prime} S(J, t), \nabla^{\prime \prime} \nabla^{\prime \prime} u\right)_{t}+2\left(\nabla^{\prime \prime} \nabla^{\prime \prime} u, \nabla^{\prime \prime} \nabla^{\prime \prime} S(J, t)\right)_{t} \\
& =2(u, L S(J, t))_{L^{2}}+2(u, \bar{L} S(J, t))_{L^{2}} .
\end{aligned}
$$

But from Lemma 2.6, we have

$$
\bar{L} S(J, t)=L S(J, t),
$$

from which the lemma follows. 
Lemma 2.8. Suppose that $(\omega, J)$ is a perturbed extremal Kähler metric and thus that the gradient vector field of $S(J, t)$ is a holomorphic vector field. If $\delta J=\nabla^{\prime \prime} \nabla^{\prime \prime} u$ for a real smooth function $u$, then

$$
(\delta L) S(J, t)=-\frac{1}{2} L\left(S(J, t)^{\alpha} u_{\alpha}-u^{\alpha} S(J, t)_{\alpha}\right)=L(\bar{L}-L) u .
$$

Proof. Recall that by Lemma 2.3 in [7]

$$
\mathcal{L}_{X} J=2 i \nabla_{J}^{\prime \prime} X^{\prime}-2 i \nabla_{J}^{\prime} X^{\prime \prime}
$$

Therefore,

$$
\begin{aligned}
\mathcal{L}_{J X} J & =2 i \nabla_{J}^{\prime \prime} i X^{\prime}-2 i \nabla_{J}^{\prime}(-i) X^{\prime \prime} \\
& =-2\left(\nabla_{J}^{\prime \prime} X^{\prime}-\nabla_{J}^{\prime} X^{\prime \prime}\right) .
\end{aligned}
$$

This shows that $\mathcal{L}_{J X} J \in T_{J} \mathcal{J}$ corresponds to $-2 \nabla^{\prime \prime} \nabla^{\prime \prime} u \in \operatorname{Sym} \otimes^{2} T^{\prime \prime *} M$ via the identification $T_{J} \mathcal{J} \cong \operatorname{Sym} \otimes^{2} T^{\prime \prime *} M$. Thus $\mathcal{L}_{-1 / 2 J X_{u}} J$ corresponds to $\nabla^{\prime \prime} \nabla^{\prime \prime} u$. On the other hand,

$$
\mathcal{L}_{\frac{1}{2} J X_{u}} \omega=d\left(i\left(\frac{1}{2} J X_{u}\right) \omega\right)
$$

and

$$
\begin{aligned}
\left(i\left(\frac{1}{2} J X_{u}\right) \omega\right)(Y) & =\omega\left(\frac{1}{2} J X_{u}, Y\right)=\omega\left(-\frac{1}{2} \operatorname{grad} u, Y\right) \\
& =\omega\left(-\frac{1}{2} X_{u}, J Y\right)=-\frac{1}{2} d u \circ J=\left(d^{c} u\right)(Y),
\end{aligned}
$$

where $d^{c}=\frac{i}{2}(\bar{\partial}-\partial)$. From (2.14) and (2.15) it follows that

$$
\mathcal{L}_{\frac{1}{2} J X_{u}} \omega=d d^{c} u=i \partial \bar{\partial} u \text {. }
$$

Let $f_{s}$ is the flow generated by $-\frac{1}{2} J X_{u}$. Suppose that $S$ is a smooth function such that $\operatorname{grad}^{\prime} S$ is a holomorphic vector field and that $\left\{S_{s}\right\}$ is a family of smooth functions parameterized by $s$ such that

$$
\operatorname{grad}_{s}^{\prime} S_{s}=\operatorname{grad}^{\prime} S, \quad \int_{M} S_{s}\left(f_{-s}^{*} \omega\right)^{m}=\int_{M} S \omega^{m},
$$

where $\operatorname{grad}_{s}^{\prime} S_{s}$ is the $(1,0)$-part of the gradient vector field of $S_{s}$ with respect to $f_{-s}^{*} \omega$. It is easy to see that if $f_{-s}^{*} \omega=\omega+i \partial \bar{\partial} \varphi$, then $S_{s}=S+S^{\alpha} \varphi_{\alpha}$. Then (2.16) shows

$$
S_{s}=S+s S^{\alpha} u_{\alpha}+O\left(s^{2}\right) .
$$

We have

$$
L\left(f_{s} J, \omega\right) f_{s}^{*} S_{s}=f_{s}^{*}\left(L\left(J, f_{-s}^{*} \omega\right) S_{s}\right)=0 .
$$


Taking the derivative of (2.18) with respect to $t$ at $t=0$, we obtain

$$
\delta L \cdot S+L\left(-\frac{1}{2}\left(J X_{u}\right) S+S^{\alpha} u_{\alpha}\right)=0 .
$$

On the other hand,

$$
\begin{aligned}
J X_{u} \cdot S & =g\left(J X_{u}, \operatorname{grad} S\right)=\omega\left(X_{u}, \operatorname{grad} S\right)=d u(\operatorname{grad} S) \\
& =(\partial u+\bar{\partial} u)\left(\nabla^{\prime} S+\nabla^{\prime \prime} S\right)=u^{\alpha} S_{\alpha}+S^{\alpha} u_{\alpha} .
\end{aligned}
$$

It follows from (2.19) and (2.20) that

$$
\begin{aligned}
\delta L \cdot S & =-L\left(-\frac{1}{2}\left(u^{\alpha} S_{\alpha}+S^{\alpha} u_{\alpha}\right)+S^{\alpha} u_{\alpha}\right) \\
& =-L\left(\frac{1}{2}\left(S^{\alpha} u_{\alpha}-u^{\alpha} S_{\alpha}\right)\right) .
\end{aligned}
$$

Applying this with $S=S(J, \omega)$ and using Lemma 2.6 complete the proof of Lemma 2.8.

Theorem 2.9. Let $J$ be a critical point of $\Phi$, i.e., $(\omega, J)$ gives a perturbed extremal Kähler metric and $u$ be a real smooth function on $M$. Then the Hessian of $\Phi$ at $J$ in the direction of $\nabla^{\prime \prime} \nabla^{\prime \prime} u$ and $\nabla^{\prime \prime} \nabla^{\prime \prime} v$ is given by

$$
\operatorname{Hess}(\Phi)_{J}\left(\nabla^{\prime \prime} \nabla^{\prime \prime} u, \nabla^{\prime \prime} \nabla^{\prime \prime} v\right)=8(u, L \bar{L} v)=8(u, \bar{L} L v) .
$$

Proof. Let $\delta J=\nabla^{\prime \prime} \nabla^{\prime \prime} v$. By using Lemma 2.7, Lemma 2.8 and Lemma 2.4 successively, one obtains

$$
\begin{aligned}
\operatorname{Hess}(\Phi)_{J}\left(\nabla^{\prime \prime} \nabla^{\prime \prime} u, \nabla^{\prime \prime} \nabla^{\prime \prime} v\right) & =4 \delta(u, L S(J, t)) \\
& =4(u, \delta L \cdot S(J, t)+L \delta S(J, t)) \\
& =4(u, L(\bar{L}-L) v+L(L+\bar{L}) v) \\
& =8(u, L \bar{L} v) .
\end{aligned}
$$

If one uses the third term in Lemma 2.7 and $\delta \bar{L}=L-\bar{L}$, then one gets the third term of Theorem 2.9. This completes the proof.

\section{Proof of Theorem 1.1}

In this section, we give a proof of Theorem 1.1. Suppose that $g$ is a perturbed extremal Kähler metric on $(M, \omega, J)$. Let $X$ be a holomorphic vector field and $\alpha$ be the dual 1 -form to $X$, that is

$$
\alpha(Y)=g(X, Y), \quad \alpha=\alpha_{\bar{i}} d \bar{z}^{i}=g_{j \bar{i}} X^{j} d \bar{z}^{i} .
$$


Since $X$ is a holomorphic vector field

$$
\bar{\partial} \alpha=\left(\nabla_{\bar{i}} \alpha_{\bar{j}}-\nabla_{\bar{j}} \alpha_{\bar{i}}\right) d \bar{z}^{i} \wedge d \bar{z}^{j}=0
$$

Let $\alpha=H \alpha+\bar{\partial} \psi$ be the harmonic decomposition, where $H \alpha$ denotes the harmonic part. Then

$$
\begin{aligned}
L \psi= & m c_{m}\left(\psi_{\bar{\ell} j k}^{i} \frac{\sqrt{-1}}{2 \pi} d z^{k} \wedge d \overline{z^{\ell}}, \omega \otimes I+\frac{\sqrt{-1}}{2 \pi} t \Theta, \ldots, \omega \otimes I+\frac{\sqrt{-1}}{2 \pi} t \Theta\right) \\
= & m c_{m}\left(\left(X^{i}-(H \alpha)^{i}\right)_{\bar{\ell} j k} \frac{\sqrt{-1}}{2 \pi} d z^{k} \wedge \overline{d z^{\ell}}, \omega \otimes I\right. \\
& \left.+\frac{\sqrt{-1}}{2 \pi} t \Theta, \ldots, \omega \otimes I+\frac{\sqrt{-1}}{2 \pi} t \Theta\right) \\
= & -m c_{m}\left((H \alpha)^{i}{ }_{\bar{\ell} j k} \frac{\sqrt{-1}}{2 \pi} d z^{k} \wedge d \overline{z^{\ell}}, \omega \otimes I\right. \\
& \left.+\frac{\sqrt{-1}}{2 \pi} t \Theta, \ldots, \omega \otimes I+\frac{\sqrt{-1}}{2 \pi} t \Theta\right) \\
= & -m c_{m}\left((H \alpha)^{i}{ }_{j k}+\left(R_{j \bar{\ell}}{ }^{i}(H \alpha)^{p}\right)_{k} \frac{\sqrt{-1}}{2 \pi} d z^{k} \wedge \overline{d \overline{z^{\ell}}, \omega \otimes I}\right. \\
& \left.+\frac{\sqrt{-1}}{2 \pi} t \Theta, \ldots, \omega \otimes I+\frac{\sqrt{-1}}{2 \pi} t \Theta\right) .
\end{aligned}
$$

Note that being $\bar{\partial}$-harmonic and being $\partial$-harmonic are equivalent on compact Kähler manifolds, and thus

$$
(H \alpha)_{\bar{q} j}=\nabla_{j}(H \alpha)_{\bar{q}}=0
$$

This implies $(H \alpha)^{i}{ }_{j}=0$. It follows that

$$
\begin{aligned}
L \psi= & -m c_{m}\left(R_{j \bar{\ell}}^{i} p, k(H \alpha)^{p} \frac{\sqrt{-1}}{2 \pi} d z^{k} \wedge d \overline{z^{\ell}}, \omega \otimes I\right. \\
& \left.+\frac{\sqrt{-1}}{2 \pi} t \Theta, \ldots, \omega \otimes I+\frac{\sqrt{-1}}{2 \pi} t \Theta\right) \\
= & -m c_{m}\left(R_{j \bar{\ell} k, p}^{i}(H \alpha)^{p} \frac{\sqrt{-1}}{2 \pi} d z^{k} \wedge d \overline{z^{\ell}}, \omega \otimes I\right. \\
& \left.+\frac{\sqrt{-1}}{2 \pi} t \Theta, \ldots, \omega \otimes I+\frac{\sqrt{-1}}{2 \pi} t \Theta\right) \\
= & -(H \alpha)^{p} \nabla_{p} S(J, t)=-(H \alpha)_{\bar{q}} \nabla^{\bar{q}} S(J, t),
\end{aligned}
$$


where we have used the second Bianchi identity $R_{j \bar{\ell}}{ }^{i} p, k=R_{j \ell}{ }_{\ell, p}^{i}$ and

$$
\begin{aligned}
\nabla_{p} S(J, t)= & \nabla_{p} \frac{1}{t}\left(c_{m}\left(\omega \otimes I+\frac{i}{2 \pi} t \Theta\right)-\omega^{m}\right) \\
= & \frac{1}{t} \nabla_{p} c_{m}\left(\omega \otimes I+\frac{i}{2 \pi} t \Theta\right) \\
= & m c_{m}\left(R_{j \bar{\ell}}{ }^{i} k, p \frac{\sqrt{-1}}{2 \pi} d z^{k} \wedge d \overline{z^{\ell}}, \omega \otimes I\right. \\
& \left.+\frac{\sqrt{-1}}{2 \pi} t \Theta, \ldots, \omega \otimes I+\frac{\sqrt{-1}}{2 \pi} t \Theta\right) .
\end{aligned}
$$

Note that $\nabla^{\bar{q}} S(J, t) \frac{\partial}{\partial \bar{z}^{q}}$ is a conjugate holomorphic vector field and that $(H \alpha)_{\bar{q}} d \bar{z}^{q}$ is a conjugate holomorphic 1 -form because $H \alpha$ is a $\partial$-harmonic $(0,1)$-form. It follows from (3.1) that $L \psi=$ constant. But since $\int_{M} L \psi \omega^{m}=$ 0 by $(2.10)$ we obtain $L \psi=0$. This implies that $\operatorname{grad}^{\prime} \psi$ is a holomorphic vector field. Then $(H \alpha)^{i} \frac{\partial}{\partial z^{i}}=X-\operatorname{grad}^{\prime} \psi$ is also holomorphic. It then follows that

$$
\nabla_{\bar{k}}(H \alpha)_{\bar{j}}=0
$$

But since $(H \alpha)$ is $\partial$-harmonic, we also have $\nabla_{k}(H \alpha)_{\bar{j}}=0$. Thus $H \alpha$ is parallel.

This proves the direct sum decomposition as a vector space

$$
\mathfrak{h}(M)=\mathfrak{a}(M)+\mathfrak{h}^{\prime}(M),
$$

where $\mathfrak{a}(M)$ is the Lie subalgebra of all parallel holomorphic vector fields and

$$
\mathfrak{h}^{\prime}(M)=\left\{X \in \mathfrak{h}(M) \mid X=\operatorname{grad}^{\prime} u \text { for some } u \in C_{\mathbb{C}}^{\infty}(M)\right\}
$$

It is easy to see

$$
\begin{gathered}
{[\mathfrak{a}(M), \mathfrak{a}(M)]=0 ;} \\
{\left[\mathfrak{a}(M), \mathfrak{h}^{\prime}(M)\right] \subset \mathfrak{h}^{\prime}(M) ;} \\
{\left[\mathfrak{h}^{\prime}(M), \mathfrak{h}^{\prime}(M)\right] \subset \mathfrak{h}^{\prime}(M) .}
\end{gathered}
$$

Now by Theorem 2.9 we have $L \bar{L}=\bar{L} L$. Thus $\bar{L}$ preserves Ker $L$. Let $E_{\lambda}$ denote the $\lambda$-eigenspace of $\left.2 \bar{L}\right|_{\operatorname{Ker} L}$. If $u \in E_{\lambda}$, then $\operatorname{grad}^{\prime} u \in \mathfrak{h}^{\prime}(M)$ and

$$
\begin{aligned}
\lambda u & =2 \bar{L} u \\
& =2(\bar{L}-L) u \\
& =S(J, t)^{\alpha} u_{\alpha}-u^{\alpha} S(J, t)_{\alpha} .
\end{aligned}
$$

This implies $\left[\operatorname{grad}^{\prime} S(J, t), \operatorname{grad}^{\prime} u\right]=\lambda \operatorname{grad}^{\prime} u$. We put

$$
\begin{gathered}
\operatorname{grad}^{\prime}\left(E_{\lambda}\right):=\mathfrak{h}_{\lambda}(M) \quad \text { for } \lambda \neq 0, \\
\operatorname{grad}^{\prime}\left(E_{0}\right):=\mathfrak{h}_{0}^{\prime}(M), \\
\mathfrak{h}_{0}=\mathfrak{a}(M)+\mathfrak{h}_{0}^{\prime}(M) .
\end{gathered}
$$


Then we obtain the decomposition

$$
\mathfrak{h}(M)=\sum_{\lambda} \mathfrak{h}_{\lambda}(M)
$$

where $\mathfrak{h}_{\lambda}(M)$ is the $\lambda$-eigenspace of $\operatorname{ad}\left(\operatorname{grad}^{\prime} S(J, t)\right)$. Note that the real and imaginary parts of an element of $\mathfrak{a}(M)$ are parallel and Killing and hence $\left[\operatorname{grad}^{\prime} S(J, t), \mathfrak{a}(M)\right]=0$.

Finally, since $E_{0}=\operatorname{Ker} L \cap \operatorname{Ker} \bar{L}$, the real and imaginary parts are respectively in $E_{0}$, that is, $E_{0}$ is the complexification of the purely imaginary functions $u$ such that $\operatorname{grad}^{\prime} u$ is holomorphic. The real parts of such grad' $u$ 's are Killing vector fields, see Lemma 2.8 in [5]. The real parts of the elements of $\mathfrak{a}(M)$ are also Killing vector fields. Thus $\mathfrak{h}_{0}(M)$ is reductive. Obviously, $\mathfrak{h}_{0}(M)$ is a maximal reductive subalgebra. This completes the proof of Theorem 1.1.

\section{References}

[1] S. Bando, An obstruction for Chern class forms to be harmonic, Kodai Math. J. 29 (2006), 337-345.

[2] E. Calabi, Extremal Kähler metrics II, in 'Differential geometry and complex analysis' (I. Chavel and H.M. Farkas, eds.), Springer-Verlag, Berlin-Heidelberg-New York, 1985, 95-114.

[3] S.K. Donaldson, Remarks on gauge theory, complex geometry and four-manifold topology, in 'Fields Medallists Lectures' (Atiyah, Iagolnitzer, eds.), World Scientific, 1997, 384-403.

[4] A. Fujiki, Moduli space of polarized algebraic manifolds and Kähler metrics, Sugaku Expositions, 5 (1992), 173-191.

[5] A. Futaki, Kähler-Einstein metrics and integral invariants, Lecture Notes in Math., 1314, Springer-Verlag, Berlin-Heidelberg-New York, 1988.

[6] A. Futaki, Stability, integral invariants and canonical Kähler metrics, Proc. Differential Geometry and its Applications, 2004, Prague, (2005), 45-58.

[7] A. Futaki, Harmonic total Chern forms and stability, Kodai Math. J. 29 (2006), 346-369, math.DG/0603706.

[8] N.C. Leung, Einstein type metrics and stability on vector bundles, J. Diff. Geom. 45 (1997), 514-546.

[9] L.-J. Wang, Hessians of the Calabi functional and the norm function, Ann. Global Anal. Geom. 29 (2) (2006), 187-196.

[10] X.-W. Wang, Moment maps, Futaki invariant and stability of projective manifolds, Comm. Anal. Geom. 12 (5) (2004), 1009-1037.

Department of Mathematics

Tokyo Institute of Technology

2-12-1, O-OKAYAMA

MEGURO

TOKYO 152-8551, JAPAN

E-mail address: futaki@math.titech.ac.jp

Received 7/10/2007, accepted 8/29/2007 